\title{
On the Information Carried by Programs about the Objects They Compute*
}

\author{
Mathieu Hoyrup ${ }^{1}$ and Cristóbal Rojas ${ }^{2}$ \\ $1 \quad$ Inria Nancy Grand Est \\ 615 rue du jardin botanique, 54600 Villers-lès-Nancy, France \\ hoyrup@inria.fr \\ 2 Departamento de Matemáticas, Universidad Andres Bello \\ República 220, Santiago, Chile. \\ crojas@mat-unab.cl
}

\begin{abstract}
In computability theory and computable analysis, finite programs can compute infinite objects. Presenting a computable object via any program for it, provides at least as much information as presenting the object itself, written on an infinite tape. What additional information do programs provide? We characterize this additional information to be any upper bound on the Kolmogorov complexity of the object. Hence we identify the exact relationship between Markov-computability and Type-2-computability. We then use this relationship to obtain several results characterizing the computational and topological structure of Markov-semidecidable sets.
\end{abstract}

1998 ACM Subject Classification F.1.1 Models of Computation/Computability theory

Keywords and phrases Markov-computable, representation, Kolmogorov complexity, Ershov topology

Digital Object Identifier 10.4230/LIPIcs.STACS.2015.447

\section{Introduction}

We assume that the reader is familiar with Turing machines and basic computability theory over the natural numbers. To define computability over infinite objects, one still uses Turing Machines but has to set up a way for them to access such objects. In any case, the input of the machine is a finite or infinite sequence of symbols written on the input tape and one has to choose a suitable way to describe infinite objects by such symbolic sequences. We now briefly describe the two main approaches that have been developed.

The first one was introduced and studied by Turing [20], Grzegorczyk [5], Lacombe [9] and later Kreitz and Weihrauch [21] and is nowadays known as Type-2-computability. In this model, the description itself is completely written on the input tape of the machine. At any time, the machine can read a finite portion of this description. We will call this the Type-2-model. The second approach, promoted by the Russian school led by Markov $[10,8]$, gives an alternative. In this model one restricts the action of the machine to operate on computable (infinite) objects only, in the sense that they have computable descriptions. Instead of having access to the description itself as in the Type-2-model, the machine here has access to a program computing a description. We will call this the Markov-model. * This work was partially supported by Inria program "Chercheur invité". CR was partially supported by
FONDECYT project 11110226 and BASAL PFB-03 CMM, Universidad de Chile.

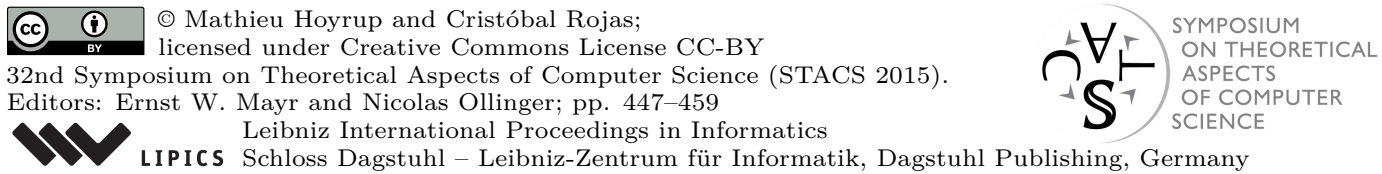


Table 1 Some celebrated results comparing Markov-computability to Type-2-computability.

\begin{tabular}{|c|c|c|}
\hline Objects & Decidability & Semidecidability \\
\hline Partial computable functions & $\begin{array}{c}\text { Markov } \equiv \text { Rice } \\
\end{array}$ & $\begin{array}{c}\text { Markov } \equiv \text { Type-2 } \\
\text { Rice-Shapiro }\end{array}$ \\
\hline Total computable functions & $\begin{array}{l}\text { Markov } \equiv \text { Type-2 } \\
\text { Kreisel et al/Ceitin }\end{array}$ & $\begin{array}{c}\text { Markov > Type- } 2 \\
\text { Friedberg }\end{array}$ \\
\hline
\end{tabular}

These two approaches provide a priori different computability notions, and their comparison has been an important subject of study [13, 11, 18, 7, 1, 4, 12, 6, 19].

It is clear that the Markov-model is at least as powerful as the Type-2-model, so the question is: does it allow to compute strictly more than the Type-2-model? The answer depends on the objects that we consider, and the algorithmic tasks we want to perform on them. The computational power of these models can therefore be classified according to these parameters. Table 1 summarizes the most celebrated results in this direction. The computable objects considered are the partial computable functions and the total computable functions. The algorithmic tasks considered are decidability and semidecidability of properties about these objects.

Kreisel-Lacombe-Shœenfield/Ceitin's Theorem [7, 1] for instance, states that over total computable functions, Markov-decidability is equivalent to Type-2-decidability ${ }^{1}$. This means that the machine trying to decide a property, when provided with a program $p$ for a function $f$, cannot do better than just running $p$ to evaluate $f$. The machine gains no additional information about $f$ from $p$. We note that Ceitin's version of this result shows that over the real line, Markov-computable functions and Type-2-computable functions coincide.

On the other hand, Friedberg [4] exhibited properties about total computable functions that are Markov-semidecidable but not Type-2-semidecidable. So that for semidecidability, a program $p$ for a function $f$ does give some additional information that can be exploited by the machine. The main question we raise in this paper is the following:

Can we characterize the additional useful information contained in a program computing an object, as compared to having the object itself?

To get some intuition, consider the following fundamental difference between the two models. In the Type-2-model, at any given time only a finite portion of the description of $x$ is provided, which corresponds to a finite approximation of $x$. Clearly, this approximation is also good for infinitely many other objects - all the ones that are "close enough" to $x$. In particular, $x$ is never completely specified. In the Markov-model on the other hand, the program provided to the machine completely specifies $x$ from the beginning of the calculation! This increases the predictive power of $M$, which might therefore be able to perform stronger calculations. The point is to understand in which situations this fact can be exploited. A trivial example is obtained when one considers the relativized setting: every function is Markov-computable relative to an appropriate (powerful enough) oracle. Whereas whatever oracle $A$ we consider, Type-2-computable functions relative to $A$ must always be continuous.

This observation takes us to another interesting point that separates the Markov-model from the Type-2-model, namely their topological structure. It is well known that Type-2computability and topology are closely related: e.g. the Type-2-computable functions are

1 In its original form, this theorem is stated for functionals. 
exactly the effectively continuous ones, and the Type-2-semidecidable properties exactly correspond to the effectively open sets. The connection between Markov-computability and topology, on the other hand, appears to be much less clear. In particular, Friedberg's construction provides a Markov-semidecidable set which is not open (for the standard topology restricted to computable elements).

An obvious solution to relate Markov-computability to topology is to consider precisely the topology generated by all the Markov-semidecidable sets - the so called Ershov's topology. The question then becomes:

How do Markov-semidecidable sets look like? can we characterize Ershov's open sets ?

In the present paper we make use of Kolmogorov Complexity to provide a fairly complete answer to these and other questions in different settings. Our main result is a characterization of the additional information provided by a program, when the class of objects considered are the computable points of an effective topological space. It can be informally stated as follows (see Section 3):

- Theorem A. Over effective topological spaces, a program computing $x$ provides as much information as a description of $x$ itself together with any upper bound on the Kolmogorov complexity of $x$.

Here, the Kolmogorov complexity $K(x)$ of a computable infinite object $x$ is to be understood as the size of the shortest program computing a description of $x$ (Kolmogorov complexity of infinite objects was first defined by Schnorr [15]). Obviously, any program for $x$ trivially provides, in addition to a description, an upper bound on its Kolmogorov complexity. Theorem A says that this bound is all the exploitable additional information it provides.

Thus, we have a third model to deal with computable infinite objects. In this model, input $x$ is presented to the machine as a pair $(d, k)$, where $d$ is a description for $x$ and $k$ a bound on the Kolmogorov complexity of $x$. We shall call this the $\boldsymbol{K}$-model. In these terms, a particular case of Theorem A can be stated as follows: if $\mathcal{X}, \mathcal{Y}$ are effective topological spaces (not necessarily metric) and $X_{c}, Y_{c}$ are the corresponding sets of computable points, then a function $f: X_{c} \rightarrow Y_{c}$ is Markov-computable if and only if it is K-computable.

A simple observation shows that one can not in general compute a program for $x$ from a K-description of $x$, meaning that the two notions are not fully equivalent. Despite this fact, Theorem A is valid in great generality: it holds for decidability, semidecidability and also higher in the hierarchy. In proving this we make a fundamental use of the Recursion Theorem. Interestingly, although the Recursion Theorem does not relativize (a well known fact), Theorem A does in many cases.

The K-model also sheds light into the structure of the open sets of Ershov's topology, providing a nice characterization in terms of Kolmogorov complexity, at least in the particular case of the extended natural numbers $\overline{\mathbb{N}}=\mathbb{N} \cup\{\infty\}$.

Theorem B. On the extended natural numbers, the Ershov topology is generated by the sets $\{n\}$ and $\{x \in \overline{\mathbb{N}}: K(x)<h(x)\}$ for some computable order $h$.

With the same techniques, we are able to prove several other related results that are interesting on their own. For example, we show that there is no effective enumeration of the Markov-semidecidable sets of $\overline{\mathbb{N}}$ and that there is a Markov-semidecidable subset of $\{0,1\}^{\mathbb{N}}$ that is not $\Sigma_{2}^{0}$.

Finally, in the search of the limitations of our techniques, we turn our attention to more general spaces and analyze functions with values on topological spaces that have an 
Table 2 Some results comparing Markov-computability, K-computability, and Type-2computability. $\mathbb{S}=\{\perp, \top\}$ is the Sierpiński space whose topology is generated by $\{\top\}$.

\begin{tabular}{|c|c|c|c|}
\hline Space $\mathcal{X}$ & Semidecidable & $\emptyset^{\prime}$-Semidecidable & F: $\mathcal{X} \rightarrow \mathcal{O}(\mathbb{B})$ \\
\hline $\mathbb{S}$ & Markov $\equiv \mathrm{K} \equiv$ Type-2 & Markov $>\mathrm{K} \equiv$ Type- 2 & Markov $>\mathrm{K} \equiv$ Type- 2 \\
\hline Partial functions & Markov $\equiv \mathrm{K} \equiv$ Type-2 & Markov $>\mathrm{K} \equiv$ Type- 2 & Markov $>\mathrm{K}>$ Type-2 \\
\hline Total functions & Markov $\equiv \mathrm{K}>$ Type-2 & Markov $\equiv \mathrm{K}>$ Type- 2 & Markov $? \mathrm{~K}>$ Type-2 \\
\hline
\end{tabular}

admissible representation but are not countably-based. In particular, when this is the space of open subsets of Baire space $\mathcal{O}(\mathbb{B})$, we show that Markov-computability can be strictly stronger than K-computability:

- Theorem C. For functions from the partial computable functions with values on $\mathcal{O}(\mathbb{B})$ one has that:

$$
\text { Markov-computability > K-computability > Type-2-computability. }
$$

One of the main question that remains open is whether the first strict inequality in Theorem $\mathrm{C}$ holds if we replace the partial computable functions by the total ones. The situation is summarized in Table 2.

The paper is organized as follows. We start by providing the basic notions and definitions in Section 2. In Section 3 we introduce the K-model and present our main results. Section 4 contains several results that shed light on the structure of Markov-semidecidable sets and in Section 5 we present the announced negative results. Finally, Section 6 contains a list of related problems for possible future work.

\section{Background}

\subsection{Notations and basic definitions.}

We assume the reader is familiar with computability theory. Let $\left\{\varphi_{e}\right\}_{e \in \mathbb{N}}$ be an effective enumeration of the set of computable partial functions. We denote by $P_{c}(\mathbb{N})$ the collection of c.e. subsets of $\mathbb{N}$ and $W_{e}=\operatorname{dom}\left(\varphi_{e}\right)$ the induced effective enumerations of its elements. If $A \in P_{c}(\mathbb{N})$, an $\boldsymbol{i n d e x}$ of $A$ is a number $e$ such that $W_{e}=A$. If $A$ is a c.e. set, implicitly given by an index, $A[s]$ is the finite subset of $A$ enumerated by stage $s$, so that $A[s] \subseteq A[s+1]$ and $A=\bigcup_{s} A[s]$. We use the notation $A[$ at $s]=A[s] \backslash A[s-1]$ if $s \geq 1$ and $A[$ at 0$]=A[0]$. If $F$ is a finite subset of $\mathbb{N}$ then $[F]$ is the collection of supersets of $F . \mathbb{B}=\mathbb{N}^{\mathbb{N}}$ will denote Baire space.

\subsection{Effective topological spaces.}

An effective topological space is a tuple $(\mathcal{X}, \tau, \mathcal{B})$ where $(\mathcal{X}, \tau)$ is a non-empty topological space, $\mathcal{B}=\left\{\mathcal{B}_{i}\right\}_{i \in \mathbb{N}}$ is numbered basis such that there exists a computable function $f$ : $\mathbb{N} \times \mathbb{N} \rightarrow \mathbb{N}$ satisfying $\mathcal{B}_{i} \cap \mathcal{B}_{j}=\bigcup_{k \in W_{f(i, j)}} \mathcal{B}_{k}$.

Given an effective topological space $\mathcal{X}$, the standard representation is defined as a surjective map $\rho: \operatorname{dom}(\rho) \subseteq \mathbb{B} \rightarrow \mathcal{X}$ satisfying $\rho(f)=x$ whenever $\{f(n): n \in \mathbb{N}\}=\{i: x \in$ $\mathcal{B}_{i}$. We will call any $f \in \rho^{-1}(x)$, a Type-2-name of $x$. An element $x$ is computable if it has a computable Type-2-name. We denote by $\boldsymbol{X}_{\boldsymbol{c}}$ the set of computable points. The countable set $X_{c}$ has a canonical numbering $\nu$ defined by $\nu(e)=x$ if $\varphi_{e}$ is a name of $x$. We 
will call such an $e$ a Markov-name of $x$. To facilitate the reading of the paper, we will use the font $A, N, U$ when working on the space $X_{c}$, and the fonts $\mathcal{A}, \mathcal{N}, \mathcal{U}$ when working on $\mathcal{X}$.

\subsubsection{Type-2-computability and Markov-computability}

Let $(\mathcal{X}, \tau, \mathcal{B})$ and $\left(\mathcal{Y}, \tau^{\prime}, \mathcal{B}^{\prime}\right)$ be effective topological spaces. In what follows $\mathrm{R}$ stands for both Type-2 and Markov. A set $A \subseteq X_{c}$ is R-semidecidable if there is a Turing machine $M$ which, when provided with an R-name of $x$, halts if and only if $x \in A$. A function $f: X_{c} \rightarrow Y_{c}$ is R-computable if there is a Turing machine $M$ which, when provided with an R-name of $x$, writes an R-name for $f(x)$ on its one-way output tape. It is not hard to see that a function $f: X_{c} \rightarrow Y_{c}$ is R-computable if and only if the sets $f^{-1}\left(\mathcal{B}_{i}^{\prime}\right)$ are uniformly R-semidecidable.

- Remark. It is worth noting that for a function $f: X_{c} \rightarrow Y_{c}$, being Markov-computable is equivalent to having a Machine $M$ which, provided with a Markov-name of $x$, outputs a Type-2-name of $f(x)$. Indeed, combining the program for $x$ with the program for $M$ gives a program for $f(x)$. We also note that a function $f: X_{c} \rightarrow Y_{c}$ which is Type-2-computable does not necessarily extend to a Type-2-computable function $\bar{f}: \mathcal{X} \rightarrow \mathcal{Y}$.

A numbering $\eta$ of $X_{c}$ is admissible if it is equivalent to the canonical numbering $\nu$ in the sense that there exists partial computable functions $f$ and $g$ such that $\nu=\eta \circ f$ on $\operatorname{dom}(\nu)$ and $\eta=\nu \circ g$ on $\operatorname{dom}(\eta)$. The Markov-computability notions do not depend on the choice of the admissible numbering. We will often use the admissible numbering $\eta$ of $X_{c}$ defined by $\eta(e)=x$ whenever $W_{e}=\left\{i \in \mathbb{N}: x \in \mathcal{B}_{i}\right\}$.

Type-2-computability and topology are closely related. A set $\mathcal{U} \subseteq \mathcal{X}$ is an effective open set if there exists $e \in \mathbb{N}$ such that $\mathcal{U}=\bigcup_{i \in W_{e}} \mathcal{B}_{i}$. If $A=\mathcal{U} \cap X_{c}$, we will then say that $A$ is effectively open $\boldsymbol{i n} \boldsymbol{X}_{\boldsymbol{c}}$. The connection is established by the following result (see [21]).

- Theorem 1. A set $A \subseteq X_{c}$ is Type-2-semidecidable if and only if it is effectively open in $X_{c}$. Therefore, a function $f: X_{c} \rightarrow Y_{c}$ is Type-2-computable if and only if it is effectively continuous, i.e. the sets $f^{-1}\left(\mathcal{B}_{i}^{\prime}\right)$ are uniformly effectively open in $X_{c}$.

As mentioned in the introduction, in order to have an analogous result for Markovcomputability, we have to use Ershov's topology on $X_{c}$, which may be different from the topology of $\mathcal{X}$ restricted to $X_{c}$.

- Example 2. Let $\mathbb{B}=\mathbb{N}^{\mathbb{N}}$ be the Baire space. For each finite sequence $u$, let $[u]$ be the set of infinite extensions of $u$, called a cylinder. We endow $\mathbb{B}$ with the topology generated by the cylinders, which is an effective topology. The standard numbering $\varphi_{e}$ of partial computable functions, restricted to the indices of total functions is an admissible numbering of $\mathbb{B}_{c}$.

- Example 3. Let $\mathcal{P}(\mathbb{N})$ be space of subsets of $\mathbb{N}$. For each finite set $F \subseteq \mathbb{N}$, let $[F]$ be the set of supersets of $F$. We endow $\mathcal{P}(\mathbb{N})$ with the Scott topology, generated by the sets $[F]$, which is an effective topology. The standard numbering $W_{e}=\operatorname{dom}\left(\varphi_{e}\right)$ of c.e. sets is an admissible numbering of $P(\mathbb{N})_{c}$.

\section{Main results}

In this section, $(\mathcal{X}, \tau, \mathcal{B})$ is always an effective topological space and $X_{c}$ is the subset of computable elements. We start by explaining the main idea behind our results. Let $x \in X_{c}$ be a fixed element. From a machine Type-2-semideciding a set $A$ containing $x$, one can 
compute a neighborhood $\mathcal{N}$ of $x$ such that for every element $y \in X_{c}$ the following implication holds:

$$
y \in \mathcal{N} \Longrightarrow y \in A .
$$

Now assume that $A$ has the weaker property of being Markov-semidecidable, and still contains $x$. From a machine Markov-semideciding $A$ one cannot in general compute such a neighborhood, which may not exist as shown by Friedberg's example. However, from the Markov-name of any other element $y \in X_{c}$ one can still compute a neighborhood $\mathcal{N}_{y}$ of $x$ such that implication (1) holds. Further, as a finite intersection of neighborhoods is still a neighborhood, one can compute a neighborhood $\mathcal{N}$ satisfying implication (1) for all $y$ in a given finite set. Using this argument we can show that the problem $x \in A$ can be Type-2-semidecided as soon as we know, in addition, a finite list of programs containing at least one for $x$. This additional information is equivalent to having any upper bound on the Kolmogorov complexity of $x$, which leads us to the notion of K-computability that we now introduce.

\subsection{K-computability}

- Definition 4. The Kolmogorov complexity $\boldsymbol{K}(\boldsymbol{x})$ of a computable element $x \in X_{c}$ is the length of a shortest program computing a Type-2-name of $x$.

In this paper, whether we use prefix-free, monotone or plain machines will not make any difference so we do not need to specify the definition any further.

- Definition 5. A $K$-name of a computable element $x \in X_{c}$ consists of a pair $(k, f)$ where $k \geq K(x)$ and $f$ is a Type-2-name of $x$.

- Remark. Note that $k$ is only an upper bound on the Kolmogorov complexity of $x$ and not necessarily of $f$, which may even be non computable. Note also that knowing any such $k$ is effectively equivalent to knowing any upper bound on a Markov-name of $x$. This is what we will rather use in our proofs.

The $\boldsymbol{K}$-computability notions are defined in the same way as in the previous section. We will denote by $\boldsymbol{X}_{\boldsymbol{c}}(\boldsymbol{k})$ the set of computable elements whose Kolmogorov complexity is at most $k$. Note that $X_{c}=\bigcup_{k} X_{c}(k)$ and that K-computability is the same as Type-2-computability on $X_{c}(k)$, uniformly in $k$. In particular, a set $A \subseteq X_{c}$ is K-semidecidable iff there exists uniformly effective open sets $\mathcal{U}_{k}$ such that $A \cap X_{c}(k)=\mathcal{U}_{k} \cap X_{c}(k)$.

Thus, for each notion of computability we have so far three versions, depending on the way the objects are represented.

It is clear that one can compute K-names from Markov-names. An important first observation is the fact that the converse does not necessarily hold. In other words, the representations underlying Markov-computability and K-computability are not equivalent.

- Proposition 6. In general, it is not possible to compute Markov-names from K-names.

One can show that on Cantor space, Markov-names are limit-computable (can be learned) from K-names: given $x$ and $k \geq K(x)$, one can compute a sequence of natural numbers converging to an index of $x$ (this problem was investigated in the context of inductive inference [3]). One can moreover show that relative to the halting set, Markov-names are uniformly computable from K-names. A c.e. set, however, cannot be learned. Actually one can prove a stronger statement. 
- Proposition 7. There is no Turing functional $\Phi$ that, given an index e and a Type-2-name of a set $W$ which is either $\mathbb{N}$ or $W_{e}$, computes a sequence of numbers converging to an index of $W$.

The rest of this section is devoted to show that, despite the facts above, the notions of Markov-computability and K-computability are indeed equivalent to a large extent.

\subsection{Equivalence between Markov-computability and K-computability}

We will use the Recursion Theorem. See [14].

- Theorem 8 (Recursion Theorem). For every computable total function $f$, there exists $e$ such that $\varphi_{e}=\varphi_{f(e)}$. Moreover, e can be computed from an index of $f$.

The following Lemma contains the main technical arguments.

- Lemma 9. Let $A$ be a c.e. subset of $\mathbb{N}$. There exist uniformly effective Scott open sets $\mathcal{U}_{k} \subseteq \mathcal{P}(\mathbb{N})$, such that for every c.e. set $E$ the following hold:

(i) if all the indices of $E$ belong to $A$ then $E \in \mathcal{U}_{k}$ for every $k$,

(ii) if no index of $E$ belongs to $A$ then $E \notin \mathcal{U}_{k}$ for every $k \geq K(E)$.

The argument is uniform: the open sets $\mathcal{U}_{k}$ are effective, uniformly in a c.e. index of $A$.

Proof. Using the Recursion theorem, there is a computable function $e(a, b)$ such that for all $a, b \in \mathbb{N}$,

$$
W_{e(a, b)}= \begin{cases}W_{a} & \text { if } e(a, b) \notin A, \\ W_{a}[t] \cup W_{b} & \text { if } e(a, b) \in A[\text { at } t] .\end{cases}
$$

Let $k \in \mathbb{N}$. We define an effective open set $\mathcal{U}_{k}$. Compute $b_{k}$ such that every element whose complexity is less than $k$ has an index less than $b_{k}$. If $a$ is such that for all $b \leq b_{k}$, $e(a, b) \in A$ then let $t$ be minimal such that $e(a, b) \in A[t]$ for all $b \leq b_{k}$, enumerate $\left[W_{a}[t]\right]$ into $\mathcal{U}_{k}$.

We now check the two announced conditions. $i$ ) Let $E \subseteq \mathbb{N}$ be a c.e. set. Assume that every index of $E$ belongs to $A$ and let $a$ be an index of $E$. For all $b, e(a, b) \in A$ (otherwise $e(a, b)$ is an index of $W_{a}=E$ but $e(a, b) \notin A$, contradiction), so $\mathcal{U}_{k}$ contains $\left[W_{a}[t]\right]$ for some $t$, which contains $E$. ii) Assume that $K(E) \leq k$, that no index of $E$ belongs to $A$ and that $E \in \mathcal{U}_{k}$. Let $b \leq b_{k}$ be an index of $E$. As $E \in \mathcal{U}_{k}, E$ belongs to some $\left[W_{a}[t]\right]$ enumerated into $\mathcal{U}_{k}$ (here $a$ is not the same as above and is not assumed to be an index of $E$ ). As $e(a, b) \in A$, $W_{e(a, b)}=W_{a}\left[t^{\prime}\right] \cup W_{b}$ for some $t^{\prime} \leq t$. As $W_{a}\left[t^{\prime}\right] \subseteq W_{a}[t] \subseteq W_{b}, e(a, b)$ is an index of $E$ that belongs to $A$, contradicting the assumption.

We now state the main explicit versions of Theorems A and B.

- Theorem 10. Let $\mathcal{X}$ be an effective topological space. A set $A \subseteq X_{c}$ is Markov-semidecidable iff it is K-semidecidable. The equivalence is uniform.

Proof. Every effective topological space is Type-2-computably homeomorphic to a subspace of $\mathcal{P}(\mathbb{N})$ : to $x \in \mathcal{X}$, associate $\left\{i \in \mathbb{N}: x \in \mathcal{B}_{i}\right\}$ where $\mathcal{B}_{i}$ is enumeration of the basis of $\mathcal{X}$. Hence we can assume that $\mathcal{X}$ is a subspace of $\mathcal{P}(\mathbb{N})$. Let $I \subseteq \mathbb{N}$ be a c.e. set such that for all $e \in \mathbb{N}$ for which $W_{e} \in X_{c}$, it holds $W_{e} \in A \Longleftrightarrow e \in I$. Each c.e. set $E \in X_{c}$ either has all its indices in $I$ or has no index in $I$, so the effective open sets $\mathcal{U}_{k}$ provided by Lemma 9 coincide with $A$ on the set of elements of $X_{c}$ whose complexity is at most $k$. Now, a machine K-semideciding $A$ works as follows: given a Type-2-name of $E \in X_{c}$ and $k \geq K(E)$, it tests whether $E \in \mathcal{U}_{k}$ and halts in this case only. 
- Corollary 11. Let $\mathcal{X}, \mathcal{Y}$ be effective topological spaces. A function $f: X_{c} \rightarrow Y_{c}$ is Markovcomputable iff $f$ is $K$-computable. The equivalence is uniform.

Proof. Let $B_{i}$ be the numbered basis of $\mathcal{Y} . \quad f$ is Markov-computable iff the sets $f^{-1}\left(\mathcal{B}_{i}\right)$ are uniformly Markov-semidecidable iff these sets are K-semidecidable (Theorem 10) iff $f$ is K-computable.

We now show that the argument in the proof of Lemma 9 can be extended from semidecidability to weaker classes of properties, showing that for most algorithmic tasks, the additional information given by programs is indeed just an upper bound on the Kolmogorov complexity.

Hierarchies. Let $\mathcal{X}$ be an effective topological space. We consider the finite levels of the effective Borel hierarchy, defined as follows. The class $\Sigma_{1}^{0}$ consists of the effective open sets. The class $\Sigma_{n+1}^{0}$ consists of the effective unions of differences of $\Sigma_{n}^{0}$-sets. The classes $\Pi_{n}^{0}$ consists of complements of $\Sigma_{n}^{0}$-sets. The class $\Delta_{n}^{0}$ is the intersection of $\Sigma_{n}^{0}$ and $\Pi_{n}^{0}$. Inside the class $\Delta_{2}^{0}$ we consider the finite levels of the effective difference hierarchy. For $n \in \mathbb{N}$, the class $\mathcal{D}_{n}$ consists of the differences of $n$ effective open sets, i.e. the sets $\left(\mathcal{U}_{0} \backslash \mathcal{U}_{1}\right) \cup \ldots\left(\mathcal{U}_{n-2} \backslash \mathcal{U}_{n-1}\right)$ if $n$ is even and the sets $\left(\mathcal{U}_{0} \backslash \mathcal{U}_{1}\right) \cup \ldots \mathcal{U}_{n-1}$ if $n$ is odd. In the case $\mathcal{X}=\mathbb{N}$ with the discrete topology, the effective Borel hierarchy is exactly the arithmetical hierarchy, the class $\mathcal{D}_{n}$ of effective difference hierarchy is exactly the class of $n$-c.e. sets.

- Theorem 12. A set $A \subseteq X_{c}$ is Markov-n-c.e. iff it is K-n-c.e. More precisely, the set of indices of elements of $A$ is $n$-c.e. on the set of indices of $X_{c}$ iff there exist uniformly effective open sets $\mathcal{U}_{k}^{1}, \ldots, \mathcal{U}_{k}^{n}$ such that $A \cap X_{c}(k)=D_{n}\left(\mathcal{U}_{k}^{1}, \ldots, \mathcal{U}_{k}^{n}\right) \cap X_{c}(k)$.

It is known from [17] that there exists a Markov-2-c.e. subset of $\mathcal{P}(\mathbb{N})$ that is not even $\Pi_{2}^{0}$. Hence Markov-2-c.e. sets are not the differences of Markov-semidecidable sets.

In the following theorem, we need to assume an additional property on the space $\mathcal{X}$. Namely, that the domain of the standard representation on $\mathcal{X}$ is a $\Pi_{2}^{0}$ set. This is the case for example for the so called quasi-Polish spaces (see [2]).

- Theorem 13. A set $A \subseteq X_{c}$ is Markov- $\Sigma_{2}^{0}$ iff it is $K$ - $\Sigma_{2}^{0}$. More precisely, the set of indices of elements of $A$ is $\Sigma_{2}^{0}$ on the set of indices of $X_{c}$ iff there exist uniformly effective open sets $\mathcal{U}_{k}^{n}, \mathcal{V}_{k}^{n}$ such that $A \cap X_{c}(k)=\bigcup_{n}\left(\mathcal{U}_{k}^{n} \backslash \mathcal{V}_{k}^{n}\right) \cap X_{c}(k)$.

- Remark. In case $\mathcal{X}$ is a Polish space, the sets $\mathcal{V}_{k}$ are not needed and therefore the last part of the statement reads $A \cap X_{c}(k)=\bigcup_{n} \mathcal{U}_{k}^{n} \cap X_{c}(k)$.

\section{Structure of Markov-semidecidable sets}

Here we provide several results that shed light on the computational and topological structure of Markov-semidecidable sets. Our first result shows that Markov-semidecidable sets share some of the nice properties of Type-2-semidecidable sets.

- Proposition 14. Assume that $\mathcal{X}$ contains a dense computable sequence. Given a Markovsemidecidable set $A$, it is semi-decidable whether $A$ is non-empty. If $A$ is non-empty, one can compute a sequence of points $\left\{x_{i}\right\} \subseteq A$ which is dense in $A$.

Proof. Using the Recursion theorem, there is a computable function $e(a)$ such that $x_{e(a)}=x_{a}$ if $e(a) \notin A$, or $x_{e(a)}$ is some point from the dense sequence in some neighborhood of $x_{a}$ if $e(a) \in A$ at time $t$. $A$ is non-empty iff there is $a$ such that $e(a) \in A$. When $A$ is non-empty, 
one can compute an element in $A$ : look for $a$ such that $e(a) \in A, x_{e(a)}$ is such a point. To get a computable dense sequence, apply this argument in parallel to the intersection of $A$ with each basic open set $\mathcal{B}_{i}$.

The following result provides an upper bound on the effective Borel complexity of Markov-semidecidable sets.

- Proposition 15. Let $A \subseteq X_{c}$ be Markov-semidecidable. There exist uniformly effective open sets $\mathcal{U}_{k} \subseteq \mathcal{X}$ such that $A=\bigcap_{k} \mathcal{U}_{k} \cap X_{c}$.

Proof. Let $\mathcal{U}_{k}$ be the effective open sets from the proof of Theorem 10 and define $\mathcal{S}=\bigcap_{k} \mathcal{U}_{k}$. We already know that $A \subseteq \mathcal{U}_{k}$ for all $k$. If $x \in X_{c} \cap \mathcal{S}$ then let $k \geq K(x)$. Since $x \in X_{c}(k) \cap \mathcal{U}_{k}=X_{c}(k) \cap A$, we conclude that $x \in A$.

The result above is actually tight, as the following theorem shows. For a finite string $u$, let us define the monotone complexity $K m(u)$ of $u$ as the length of a shortest program computing a (finite or infinite) binary sequence extending $u$. The program writes its output on a one-way output tape and may never halt. Again the precise definition of $K m(u)$ (Levin or Schnorr monotone or process complexity) does not make any difference for our purposes. The only important property is that for a computable sequence $x, K m\left(x \uparrow_{n}\right) \leq K m(x)$ for all $n$. For the sake of completeness, let us recall original Friedberg's example. We present it in a way that is more convenient for our purposes.

- Theorem 16 (Friedberg). On the Cantor space, the set

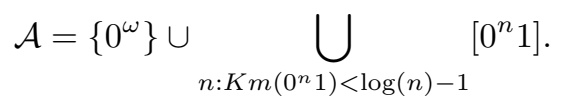

is Markov-semidecidable but not open. Hence the Ershov topology is strictly stronger than the Cantor topology.

Proof. We show that $\mathcal{A}$ is $\mathrm{K}$-semidecidable. Given an infinite binary sequence $x$ (a Type-2description) and a bound $k$ on $K(x)$, we only need to read the first $e=2^{k+2}$ bits of $x$. If we see only zeros, we accept. Otherwise one gets $0^{n} 1 \ldots$ for some $n<e$, then test whether $K m\left(0^{n} 1\right)<\log (n)-1$.

- Remark. Friedberg's example happens to be $\Sigma_{2}^{0}$. It is an effective open set appended with a limit point. We strengthen Friedberg's example by constructing a Markov-semidecidable set which is far from being open.

- Theorem 17. There is a Markov-semidecidable subset of $\{0,1\}_{c}^{\mathbb{N}}$ that is not $\Sigma_{2}^{0}$. It is a non-empty closed subset of $\{0,1\}_{c}^{\mathbb{N}}$ with empty interior, defined by

$$
A=\left\{x \in\{0,1\}_{c}^{\mathbb{N}}: \forall n, K m\left(x \uparrow_{n}\right)<n / 2+c\right\} \quad \text { for some sufficiently large } c \in \mathbb{N} .
$$

For the following results, we restrict our attention to the space $\mathcal{X}=\overline{\mathbb{N}}=\mathbb{N} \cup\{\infty\}$ whose topology is generated by the singletons $\{n\}$ and the semi-lines $[n, \infty]$, for $n \in \mathbb{N}$. Note that every point in this space is computable, so that $\mathcal{X}=X_{c}$.

Friedberg's example translated to this space reads $\{x \in \overline{\mathbb{N}}: K(x)<\log (x)-1\}$, which inspires the following definition.

- Definition 18. We define the Friedberg sets of $\overline{\mathbb{N}}$ to be the ones of the form $\{x \in \overline{\mathbb{N}}$ : $K(x)<h(x)\}$, where $h: \mathbb{N} \rightarrow \mathbb{N}$ is any computable order, namely, any non decreasing unbounded computable function. 
Note that a computable order can always be extended to a computable function $h: \overline{\mathbb{N}} \rightarrow \overline{\mathbb{N}}$, with $h(\infty)=\infty$.

Friedberg sets are Markov-semidecidable just like the original set. The next two results show that, unlike Cantor space, the only Markov-semidecidable sets over $\overline{\mathbb{N}}$ which are not Type-2-semidecidable are essentially the Friedberg sets.

- Proposition 19. If $A \subseteq \overline{\mathbb{N}}$ is Markov-semidecidable and contains $\infty$ then there is a computable order $h$ such that A contains a Friedberg set.

Proof. Since $A$ is K-semidecidable, for each $k$ one can compute $p(k)$ such that $[p(k), \infty] \cap\{x$ : $K(x) \leq k\} \subseteq A$. One can assume that $p(k)$ is increasing. Let $h(n)=\min \{i: p(i)>n\}$. If $n \notin A$ then $p(K(n))>n$ (just take $k=K(n))$, so one has $h(n) \leq K(n)$.

- Remark. Observe that $K(x)$ here coincides with the usual notion of Kolmogorov complexity of natural numbers.

Proposition 19 provides a nice characterization of the Ershov's open sets.

- Corollary 20. The Ershov topology is generated by the singletons $\{n\}$ and the Friedberg sets.

- Remark. Note that the sets $[n, \infty]$ can be expressed as the Friedberg sets $\{x \in \overline{\mathbb{N}}: K(x)<$ $h(x)\}$ where $h(x)=0$ for $x<n$ and $h(x)=c(x+1)$ for $x \geq n$, where $c$ is such that $K(n) \leq c(n+1)$ for all $n \in \mathbb{N}$.

Whether or not one can find such a characterization on other spaces such as the Cantor space is an interesting question.

We end this section by observing that, unlike Type-2-semidecidable sets, Markovsemidecidable sets cannot be effectively enumerated.

- Proposition 21. There is no effective enumeration of the Markov-semidecidable subsets of $\overline{\mathbb{N}}$.

\section{When Markov beats Kolmogorov}

In this section we explore the limits of our methods. We first look at the relativized case, and show that there are simple cases that separate Markov-computability from K-computability. However, we also show that, interestingly, the equivalence persists if the space has a Polish structure.

\subsection{Relativization}

Let $\mathbb{S}=\{\perp, \top\}$ be the Sierpiński space with topology given by $\{\emptyset,\{\top\},\{\perp, \top\}\}$. Note that as $\mathbb{S}$ is finite, K-computability is trivially equivalent to Type-2-computability simply because all the elements share a common upper bound on their Kolmogorov complexities, which therefore provides no interesting information. Relativizing w.r.t. the Halting set, we can then separate Markov-decidability from Type-2-decidability, and therefore from K-decidability.

- Remark 22. The set $\{\perp\} \subseteq \mathbb{S}$ is Markov-decidable relative to the Halting set but is not Type-2-decidable relative to any oracle.

Proof. It is not decidable relative to any oracle simply because it is not clopen. 
Similarly, over $\mathcal{P}(\mathbb{N})$, $\emptyset^{\prime \prime}$ separates K-semidecidability from Type-2-semidecidability (without oracle, the two notions coincide with Markov-semidecidability by Rice-Shapiro theorem).

- Proposition 23. The set $\{\mathbb{N}\} \subseteq \mathcal{P}(\mathbb{N})$ is K-semidecidable relative to $\emptyset^{\prime \prime}$ but is not Type-2semidecidable relative to any oracle.

However, metric spaces behave differently. Although stated on Cantor space, the next result extends to any computable metric space [21].

- Proposition 24. Let $O \subseteq \mathbb{N}$. A subset of $\{0,1\}_{c}^{\mathbb{N}}$ is Markov-semidecidable relative to $O$ if and only if it is $K$-semidecidable relative to $O$.

Proof. There are two cases, depending on whether $O$ computes the halting set or not.

If $O$ computes the halting set then by the remark following Proposition 6, Markov-names can be uniformly computed from $\mathrm{K}$-names relative to $O$, which gives the result. This part only works in the case of the Cantor space.

If $O$ does not compute the halting set then we show that Lemma 9 and Theorem 10 still hold relative to $O$ on any effective topological space. We show an alternative proof of Lemma 9 avoiding the Recursion theorem. There is a computable function $e(a, b, c)$ such that

$$
W_{e(a, b, c)}= \begin{cases}W_{a} & \text { if } \varphi_{c}(c) \text { does not halt, } \\ W_{a}[t] \cup W_{b} & \text { if } \varphi_{c}(c) \text { halts in time } t .\end{cases}
$$

Let $A \subseteq \mathbb{N}$ be c.e. relative to $O$. Given $k \in \mathbb{N}$ we define an effective open set $\mathcal{U}_{k}$. Compute $b_{k}$ such that every element whose complexity is less than $k$ has an index less than $b_{k}$. If $a$ is such that for all $b \leq b_{k}$, there exists $c=c(a, b)$ such that $e(a, b, c) \in A$ and $\varphi_{c}(c)$ halts then let $t$ be the minimal halting time of $\varphi_{c}(c)$ for $c=c(a, b)$ with $b \leq b_{k}$.

By the same argument as in the proof of Lemma 9, if no index of $E$ belongs to $A$ then $E \notin \mathcal{U}_{k}$ for all $k \geq K(E)$. If all the indices of $E \subseteq \mathbb{N}$ belong to $A$ but $E \notin \mathcal{U}_{k}$ for some $k$ then let $a$ be an index of $E$. For each $b, c$, if $\varphi_{c}(c)$ does not halt then $W_{e(a, b, c)}=W_{a}=E$ so $e(a, b, c) \in A$. As $E \notin \mathcal{U}_{k}$, there is $b \leq b_{k}$ such that for all $c$, if $\varphi_{c}(c)$ halts then $e(a, b, c) \notin A$. As a result, for all $c, \varphi_{c}(c)$ halts iff $e(a, b, c) \notin A$, so the complement of the halting set is many-one reducible to $A$ so it is Turing reducible to $O$, a contradiction.

\subsection{Functions to non-effective topological spaces}

In this section we provide results that strictly separate our three notions of computability: Markov-computability, K-computability and Type-2-computability. The idea of the constructions is to build uniform versions of the examples given in Remark 22 and Proposition 23. For this, one can imagine a function with two arguments, where the second argument $f \in \mathbb{B}$ is always provided to the machine by a Type-2-name and plays the role of the oracle. The only difficulty is then to make the computation work in a uniform way in the oracle parameter. In order to get a well defined function w.r.t. our models, we express it as a function of the first argument only, but with values on $\mathcal{O}(\mathbb{B})$, which is the set of open subsets of the Baire space endowed with the topology generated by the following sets: given a compact set $K \subseteq \mathbb{B}$, the class of open subsets of $\mathbb{B}$ containing $K$ is open. This topology is not countably-based, and hence it is not an effective topology. However it does have an admissible representation [16].

We now present the details of the simplest case, a uniform version of Remark 22 . This result contrasts with Corollary 11. 
- Theorem 25. There exists a Markov-computable function $F: \mathbb{S} \rightarrow \mathcal{O}(\mathbb{B})$ that is not K-computable.

Proof. We use the admissible numbering $\nu_{\mathbb{S}}$ of $\mathbb{S}$ defined by $\nu_{\mathbb{S}}(e)=\top$ if $\varphi_{e}(e) \downarrow, \nu_{\mathbb{S}}(e)=\perp$ otherwise. We define two effective open sets $U_{\perp}, U_{\top}$ and define $F(\perp)=U_{\perp}$ and $F(\top)=U_{\top}$. First, let $U_{\perp}=\mathbb{B}$. Let $T: \mathbb{N} \rightarrow \mathbb{N}$ be defined as follows: $T(n)$ is the halting time of $\varphi_{n}(n)$ if it halts, $T(n)=0$ otherwise. The open set $U_{\top}:=\mathbb{B} \backslash\{T\}$ happens to be effective. First the function $F$ is not Lacombe computable because it is not continuous: indeed, $F$ is not monotonic as $\perp \leq \top$ but $U_{\perp}=\mathbb{B}$ is not contained in $U_{\top} \subsetneq \mathbb{B}$. As $\mathbb{S}$ is finite, $F$ is not K-computable neither. However $F$ is Markov-computable. Given an index $e$ of $s \in \mathbb{S}$, enumerate $U_{T}$ and enumerate the set of functions $f$ such that $\varphi_{e}(e)$ does not halt in exactly $f(e)$ steps. The latter set of functions is effectively open, uniformly in $e$. If $\varphi_{e}(e) \uparrow$ then the whole space $\mathbb{B}$ is enumerated. If $\varphi_{e}(e) \downarrow$ then nothing more than $U_{\mathrm{T}}$ is enumerated. Intuitively, given $e$ and $f$, from $T$ one can decide whether $\varphi_{e}(e)$ halts, i.e. whether $\nu_{\mathbb{S}}(e)=\perp$.

A similar construction, based on Proposition 23, yields a function $F: \mathcal{P}(\mathbb{N}) \rightarrow \mathcal{O}(\mathbb{B})$ which is K-computable but not Type-2-computable by replacing the function $T$ from Theorem 25 by a function $T^{\prime}$ computing $\emptyset^{\prime \prime}$ and such that $\mathbb{B} \backslash\left\{T^{\prime}\right\}$ is effectively open.

Combining all these results, and using that fact that Theorem 25 can clearly also be realized using $\mathcal{P}(\mathbb{N})$ in place of $\mathbb{S}$, we obtain our announced Theorem $\mathrm{C}$.

- Theorem 26. For functions from $\mathcal{P}(\mathbb{N})$ with values on $\mathcal{O}(\mathbb{B})$ one has that:

Markov-computability $>$ K-computability $>$ Type-2-computability.

While Type-2-computable functions are always Scott continuous (i.e. monotone and compact), one can show that K-computable functions are always monotone but not necessarily compact. Markov-computable functions may even not be monotone.

Let us now briefly discuss whether Theorem 26 holds for functions from the Cantor space to $\mathcal{O}(\mathbb{B})$. Friedberg's example of a Markov (hence K)-semidecidable set that is not Type-2-semidecidable directly implies the second inequality. However the idea behind the proof of the first inequality cannot be applied on Cantor space. Indeed, using Proposition 24 one can show that the analog of the function of Theorem 25 is actually K-computable.

- Proposition 27. The function $G:\{0,1\}^{\mathbb{N}} \rightarrow \mathcal{O}(\mathbb{B})$ mapping $0^{\omega}$ to $\mathbb{B}$ and any other sequence to $\mathbb{B} \backslash\{T\}$ is K-computable.

Proof. Given $x, k$ and $f$, apply the algorithm given by Proposition 24 to semi-decide, if $f=T$, whether $x=0^{\omega}$. In parallel, semidecide whether $f \neq T$.

We leave the following question open: is there a Markov-computable function from the Cantor space to $\mathcal{O}(\mathbb{B})$ that is not K-computable?

\section{$6 \quad$ Future work}

We list a few problems for future work.

- Find a characterization of the Ershov topology on other spaces than $\overline{\mathbb{N}}$, like the Cantor space.

- Determine for which levels of the effective difference hierarchy the Markov-model and the $\mathrm{K}$-model are equivalent. We know from Theorem 12 that the equivalence holds for the finite levels. What about the level $\omega$ ? 
- All our results hold when the space $\mathcal{X}$ is an effective topological space. However the three models also make sense on any represented space. It seems like an interesting research program to study the extent to which our results are valid in this case.

- Compare the effective Borel hierarchy induced by the Markov-semidecidable sets, the hierarchy induced by the arithmetical hierarchy on the indices and the effective Borel hierarchy induced by the standard topology.

\section{References}

1 G. S. Ceitin. Algorithmic operators in constructive metric spaces. Trudy Matematiki Instituta Steklov, 67:295-361, 1962. English translation: American Mathematical Society Translations, series 2, 64:1-80, 1967.

2 Matthew de Brecht. Quasi-polish spaces. Ann. Pure Appl. Logic, 164(3):356-381, 2013.

3 Rusins Freivalds and Rolf Wiehagen. Inductive inference with additional information. Journal of Information Processing and Cybernetics, 15:179-185, 1979.

4 Richard M. Friedberg. Un contre-exemple relatif aux fonctionnelles récursives. Comptes Rendus de l'Académie des Sciences, 247:852-854, 1958.

5 Andrzej Grzegorczyk. On the definitions of computable real continuous functions. Fundamenta Mathematicae, 44:61-71, 1957.

6 Peter Hertling. Computable real functions: Type 1 computability versus Type 2 computability. In $C C A, 1996$.

7 G. Kreisel, D. Lacombe, and J.R. Schœnfield. Fonctionnelles récursivement définissables et fonctionnelles récursives. Comptes Rendus de l'Académie des Sciences, 245:399-402, 1957.

8 Boris A. Kushner. The constructive mathematics of A. A. Markov. Amer. Math. Monthly, 113(6):559-566, 2006.

9 Daniel Lacombe. Extension de la notion de fonction récursive aux fonctions d?une ou plusieurs variables réelles I-III. Comptes Rendus Académie des Sciences Paris, 240,241:2478-2480,13-14,151-153, 1955.

10 A. A. Markov. On the continuity of constructive functions (russian). Uspekhi Mat. Nauk, 9:226-230, 1954.

11 J. Myhill and J. C. Shepherdson. Effective operations on partial recursive functions. Mathematical Logic Quarterly, 1(4):310-317, 1955.

12 Marian B. Pour-El. A comparison of five "computable" operators. Mathematical Logic Quarterly, 6(15-22):325-340, 1960.

13 H. G. Rice. Classes of recursively enumerable sets and their decision problems. Transactions of the American Mathematical Society, 74(2):pp. 358-366, 1953.

14 Hartley Jr. Rogers. Theory of Recursive Functions and Effective Computability. MIT Press, Cambridge, MA, USA, 1987.

15 C.P. Schnorr. Optimal enumerations and optimal gödel numberings. Mathematical systems theory, 8(2):182-191, 1974.

16 Matthias Schröder. Extended admissibility. Theoretical Computer Science, 284(2):519-538, 2002 .

17 Victor L. Selivanov. Index sets in the hyperarithmetical hierarchy. Siberian Mathematical Journal, 25:474-488, 1984.

18 N. Shapiro. Degrees of computability. Transactions of the American Mathematical Society, 82:281-299, 1956.

19 Dieter Spreen. Representations versus numberings: on the relationship of two computability notions. Theoretical Computer Science, 262(1):473-499, 2001.

20 Alan Turing. On computable numbers, with an application to the Entscheidungsproblem. Proceedings of the London Mathematical Society, 2, 42:230-265, 1936.

21 Klaus Weihrauch. Computable Analysis. Springer, Berlin, 2000. 